\title{
Outcome of Evidence-based Allocation of Single-dose Antibiotic extended to Three-dose Antibiotic Prophylaxis in Surgical Site Infection
}

\author{
${ }^{1}$ Sangita D Jogdand, ${ }^{2}$ Raju K Shinde, ${ }^{3}$ Naman Chandrakar
}

\begin{abstract}
Aim: To study the outcome of evidence-based allocation of single-dose antibiotic extended to three-dose antibiotics prophylaxis in surgical site infection (SSI).

Materials and methods: A total of 183 clean surgical procedures in all age groups and of both genders with encountered comorbidities were included in the study. Surgical procedures like inguinal hernia, primary vaginal hydrocele, congenital hernia and hydrocele, fibroadenoma, and other surface swellings were targeted procedures. These procedures were of $<1$ hour duration and American Society of Anesthesiologists (ASA) grades I and II in study population. Ceftriaxone with sulbactam $1.5 \mathrm{gm}$ was prophylactic antibiotic given 1 hour prior to incision. Excess local signs for inflammation were observed strictly for extension of antibiotics to three doses or multiple doses.
\end{abstract}

Results: Success of evidence-based policy of randomization to single or three doses was $76.5 \%$ in all patients, and converting to three doses was $85.7 \%$. Favorable outcome was seen in the patients with comorbidities-76.19\% in hypertension, $57.14 \%$ in diabetes, and $28.57 \%$ in obesity. Only $14.3 \%$ of overall patients required extended multiple dose antibiotic therapy, which clearly projects that evidence-based policy implementation was effective in reducing number of doses.

Conclusion: Evidence-based flexible antibiotic dose is effective in commonly performed procedures even with comorbidities. Flexibility depending on local signs to modify dose policy gives piece of mind with excellent outcome.

Clinical significance: Adopting flexible antibiotic dosing reduces cost of antibiotics therapy with positive mindset for accepting reduced numbers of doses without affecting the outcome of surgical procedure.

Keywords: Dose policy, Evidence-based randomization, Surgical site infection.

${ }^{1}$ Associate Professor, ${ }^{2}$ Professor and Head, ${ }^{3}$ Senior Resident

${ }^{1}$ Department of Pharmacology, Jawaharlal Nehru Medical College, Wardha, Maharashtra, India

2Department of General Surgery, Jawaharlal Nehru Medical College, Wardha, Maharashtra, India

${ }^{3}$ Department of Surgery, Jawaharlal Nehru Medical College Wardha, Maharashtra, India

Corresponding Author: Raju K Shinde, AF-13, Acharya Vinoba Bhave Hospital Campus, Sawangi Meghe, Wardha Maharashtra, India, Phone: +918087148087 , e-mail: raju. shinde95@gmail.com
How to cite this article: Jogdand SD, Shinde RK, Chandrakar N. Outcome of Evidence-based Allocation of Single-dose Antibiotic extended to Three-dose Antibiotic Prophylaxis in Surgical Site Infection. Int J Recent Surg Med Sci 2017;3(2):79-84.

Source of support: Nil

Conflict of interest: None

\section{INTRODUCTION}

There are guidelines for prevention of SSI from different centers of countries. Different antibiotics or combinations are commonly used for prophylaxis and mostly misused for long duration. ${ }^{1-3}$ Operative wounds are prone to infections, most commonly due to nosocomial bacterial infection, which adds morbidity and mortality to procedure. Surgical site infection varies from institute to institute worldwide. Increased rate of SSI is noted in India compared with Western world and may be related to excess burden of patients or other antisepsis-related causes. Staphylococcus aureus, Pseudomonas aeruginosa, Escherichia coli, and Klebsiella pneumonia are common infecting microorganisms. Among these, S. aureus is the most consistent SSI-related bacteria. ${ }^{4}$

Organisms isolated from surgical wounds have different antibiotic sensitivity, or multidrug resistance may be related to inappropriate use of antibiotics, infections due to beta-lactamase producing organisms, organism having resistance to methicillin, or biofilm production by infecting organisms. ${ }^{5}$

Comorbid conditions like diabetes mellitus and obesity are the major risk factors for increase in SSI; increased preoperative hospital stay and even smoking adds to increased rate of SSI. Prevention of SSI depends on selection of chemoprophylactic agents as it is specific for particular systemic procedures. ${ }^{6}$

Surgical site infection is multifactorial, ranging from host defense, virulence of infecting organism, site of operation to entering of a system like gastrointestinal tract (GIT), biliary tract, or urinary tract. Increased rate of infection is also associated with extent of surgical dissection, seroma or hematoma formation, and presence of necrotic material in clean wound due to inappropriate use of energy devices; hence, empirical antibiotic prophylaxis 
should target the most common infecting organism for that operative procedure.

Rational for evidence-based antibiotics policy (flexible dose policy) is that even with appropriate antibiotics use as per hospital antibiotics policy, SSI is reduced but not eliminated. In India, mostly combination chemotherapy is used for prophylaxis and continued for 5 to 7 days to limit surgical wound infection. These extended duration of therapy imposes financial burden to patient or government without any additional benefit to patient, which ultimately leads to wastage of resources. Researchers have clearly depicted that single-dose or three-dose antibiotics are effective in controlling SSIs, but considering the aseptic conditions and workload in operating room, there is limited mindset of treating Indian surgeons to adhere with Western guidelines.

Rather than following strict dosing pattern, flexible dosing depending on the evidence of likely infection-if dosing is modified-will give peace of mind to treating surgeons and patient would be in less financial burden. Hence, the present study was undertaken to assess the outcome of the single-dose antibiotics therapy extended to three-dose antibiotic ( 24 hours) therapy, irrespective of patient-related comorbidities or hospital-associated factors.

\section{MATERIALS AND METHODS}

Evidence-based randomized prospective study of single dose or extended three doses were conducted at Jawaharlal Nehru Medical College from October 2013 to August 2016. Patients of common surgical procedures of all age groups of planned clean surgeries lasting $<1$ hour duration were included in this study. Patients were assessed for any comorbid conditions and controlled to optimal level preoperatively. Ceftriaxone and sulbactam in fixed combination of 2:1 were used. Dose of antibiotics was modified in pediatric age group patients according to weight of the patient. Operative site was prepared just before surgery and skin was prepared with $10 \%$ povidone iodine. Polygalactine 910 (Vicryl) was used for tissue approximation and skin was closed with nylon or subcuticular suture using absorbable suture Vicryl. Postoperatively, vital signs and fever were monitored.

\section{Patient's Inclusion Criterion}

Clean surgeries, i.e., any operative procedure where no system is being entered (like GIT, respiratory, or urinary system) of anterior abdominal wall, scrotum, breast, and other sites.

\section{Elective List Patients with ASA Grades I and II}

Patients of both genders of all age groups and comorbidities like diabetes mellitus, hypertension and obesity. Surgeries lasting $<1$ hour duration.

\section{EVIDENCE-BASED RANDOMIZATION}

\section{Criterion for Single-dose Antibiotics}

All patients with optimal tissue handling without any breach in sterile techniques were selected for a single dose of ceftriaxone $1 \mathrm{gm}$ and sulbactam $0.5 \mathrm{gm}$ intravenously 1 hour prior to induction of anesthesia. Patients who had postoperative continued fever more than $100^{\circ} \mathrm{F}$, local erythema, or increased pain than expected, single-dose administration were extended to three doses.

\section{Criteria for Three-dose Antibiotics}

Patients with excess tissue handling where chances hematoma or seroma formation was expected were primarily selected for three doses antibiotic therapy.

\section{Extension of Antibiotics Therapy}

In both groups incision site was inspected after 24 hours. If incision site was healthy without any local evident complications, antibiotics was stopped and in those cases where symptoms were not resolved, local erythema persists, signs of local collection, or pus discharge, antibiotic administration was extended or changed as per sensitivity of microorganism. Outcome of number of doses related to antibiotic policy was assessed based on the required number of patients converted to multidose therapy.

\section{DRUG USED}

Fixed combination of ceftriaxone and sulbactam (1:0.5) was used for prevention of SSI in evidence-based randomization policy. ${ }^{8}$

\section{RESULTS}

A total of 183 surgical procedures were included in the present study, of which 122 patients $(66.7 \%)$ were categorized to single dose, whereas 61 patients $(33.3 \%)$ were categorized to three-dose antibiotic regime. Patients of all age group ranging between 2 and 85 years (mean age 43.66 \pm 18.98 years) were included in the present study (Table 1 ).

Table 1: Distribution of patients according to age

\begin{tabular}{lll}
\hline Age in years & Number of patients & Percentage \\
\hline Up to 10 & 13 & 7.10 \\
$11-20$ & 12 & 6.56 \\
$21-30$ & 26 & 14.21 \\
$31-40$ & 24 & 13.11 \\
$41-50$ & 36 & 19.67 \\
$51-60$ & 37 & 20.22 \\
$>60$ & 35 & 19.13 \\
\hline Total & 183 & 100 \\
\hline Mean \pm SD & $43.66 \pm 18.98(2-85$ years $)$ &
\end{tabular}

SD: Standard deviation 


\section{Dose Effectiveness}

Out of 122 patients categorized to single-dose antibiotic therapy depending on extent of tissue dissection, $89(72 \%)$ patients were managed successfully by single-dose antibiotic therapy, whereas 17 patients required extension of antibiotic therapy to three doses and 16 patients required further extension of multiple doses.

Out of 61 patients categorized to three-dose category randomized based on more extensive dissection in cases of more adhesions, larger tumor, only 10 patients required multidose therapy, i.e., compliance was $83.6 \%$.

Success of evidence-based policy of randomization to single or three doses was $76.5 \%$ in all patients, and extension policy to three doses was $85.7 \%$.

In the present series, $26(14.2 \%)$ patients were not complied to set policy due to excess local symptoms or complications ranging from formation seroma to wound dehiscence (Table 2).

\section{Spectrum of Surgical Procedures and Outcome}

Procedures included in the present study were eversion of sac for primary vaginal hydrocele (33.3\%), followed by adult inguinal hernia mesh repair (31.7\%), other surgeries like pediatric hernia or hydrocele, fibroadenoma breast, epigastria hernia, unruptured dermoid cyst, and subcutaneous lipomas.

Table 2: Outcome of single-dose and three-dose antibiotic group

\begin{tabular}{lllll}
\hline & $\begin{array}{l}\text { Number } \\
\text { of }\end{array}$ & $\begin{array}{l}\text { Effectiveness } \\
\text { Ext. to }\end{array}$ & $\begin{array}{l}\text { Ext. to } \\
\text { multiple } \\
\text { three dose }\end{array}$ \\
dose
\end{tabular}

Nine patients developed minor local complications: four from single-dose group and five from three-dose group that does not fulfil the criterion of SSI treated with extended duration so as to avoid SSIs.

Seventeen patients- 9 from single dose and 8 patients from three doses-were positive for microorganisms representing the SSI rate of $9.2 \%$, which were treated according to sensitivity to antibiotic (Table 3).

\section{Associated Comorbidities and Outcome}

Comorbidities like diabetes mellitus $(8.7 \%)$, systemic hypertension $(8.7 \%)$, diabetes with hypertension $(2.7 \%)$, and obesity $(3.8 \%)$ were associated with the different surgical cases. Diabetes and obesity were associated with increased number of SSI.

Out of the 21 diabetic patients, 12 patients complied with our policy of single dose extended to three-dose regime; other 9 patients required to continue antibiotics or modify as per sensitivity of the organism.

Out of 21 patients with hypertension, only 5 patients required extension of antibiotics to multiple doses. Effectively, more than $75 \%$ of patient from this group saved from increased economical burden.

A total of 7 patients were recruited in obesity cohort. Only 2 out of 5 patients who received single-dose therapy could be satisfactorily managed. Three patients of single-dose therapy and all from three-dose therapy, i.e., 2 patients, were required to extend multiple drug doses (Table 4).

\section{DISCUSSION}

All surgical procedures in our study are related to soft tissue only, having no entry to respiratory, gastrointestinal, urinary, or biliary system. The most common infecting organism reported by most of the studies is

Table 3: Effectiveness of antibiotic dose policy in different surgical procedures

\begin{tabular}{|c|c|c|c|c|}
\hline Percentage of surgical procedure & Dose policy & Effective & Ext. to three doses & Ext. to multiple doses \\
\hline \multirow[t]{3}{*}{ Primary vaginal hydrocele $33.3 \%$} & Single dose $N=43$ & 35 & 6 & 2 \\
\hline & Three doses $\mathrm{N}=18$ & 14 & - & 4 \\
\hline & Total $\mathrm{N}=61$ & 49 & 6 & 6 \\
\hline \multirow[t]{3}{*}{ Adult hernia $31.7 \%$} & Single dose $N=39$ & 27 & 5 & 7 \\
\hline & Three doses $\mathrm{N}=19$ & 16 & - & 3 \\
\hline & Total $\mathrm{N}=58$ & 43 & 5 & 10 \\
\hline \multirow[t]{3}{*}{ Congenital hernia/hydrocele $6.0 \%$} & Single dose $N=7$ & 7 & 0 & 0 \\
\hline & Three doses $\mathrm{N}=4$ & 3 & - & 1 \\
\hline & Total $\mathrm{N}=11$ & 10 & - & 1 \\
\hline \multirow[t]{3}{*}{ Fibroadenoma $8.7 \%$} & Single dose $N=12$ & 6 & 3 & 3 \\
\hline & Three doses $\mathrm{N}=4$ & 3 & - & 1 \\
\hline & Total $\mathrm{N}=16$ & 9 & 3 & 4 \\
\hline \multirow[t]{3}{*}{ Other procedures $20.3 \%$} & Single dose $N=21$ & 14 & 3 & 4 \\
\hline & Three doses $\mathrm{N}=16$ & 15 & - & 1 \\
\hline & Total $\mathrm{N}=37$ & 29 & 3 & 5 \\
\hline
\end{tabular}


Table 4: Effectiveness of antibiotics policy in different comorbidities

\begin{tabular}{|c|c|c|c|c|}
\hline Comorbidity & Dose policy & Effective & Ext. to three doses & Ext. to multiple doses \\
\hline \multirow[t]{3}{*}{ Diabetes mellitus } & Single dose $\mathrm{N}=11$ & 4 & 1 & 6 \\
\hline & Three doses $N=10$ & 7 & - & 3 \\
\hline & Total N = $21(11.48 \%)$ & 11 & 1 & 9 \\
\hline \multirow[t]{3}{*}{ Hypertension } & Single dose $N=11$ & 5 & 4 & 2 \\
\hline & Three doses $N=10$ & 7 & - & 3 \\
\hline & Total $\mathrm{N}=21(11.48 \%)$ & 12 & 4 & 5 \\
\hline \multirow[t]{3}{*}{ Obesity } & Single dose $N=5$ & 2 & - & 3 \\
\hline & Three doses $\mathrm{N}=2$ & - & - & 2 \\
\hline & Total $\mathrm{N}=07(3.83 \%)$ & 2 & - & 5 \\
\hline
\end{tabular}

S. aureus, which may be due to predominant of the resident commensal of skin flora, while the other common organisms are Pseudomonas, Klebsiella, E. coli, and Proteus with varied frequencies, which warrants appropriate antibiotics prophylaxis. ${ }^{4}$ In the current scenario, these organisms have developed resistance to oxacillin/methicillin, and are found increasingly in SSI nationally and internationally. Resistance is attributed to beta-lactamase enzyme and biofilm production. Surgical site infection due to resistant organism needs proper selection of antibiotic. The most effective antibiotics against these organisms are vancomycin, linezolid, and aminoglycosides but should be considered as reserved antibiotics to minimize misuse and preferred to resistant or nosocomial infection by S. aureus. These drugs also have higher toxicity, hence their use should be limited. ${ }^{5,8}$ Due to irrational use of antibiotics, bacteria developed resistance to extendedspectrum penicillin; hence, combining sulbactam/ tazobactam with extended spectrum penicillin or cephalosporin may be helpful to stabilize beta-lactam ring and increase spectrum of activity as well as avoid development of resistance and limiting the SSI. ${ }^{9}$

\section{Effectiveness of Dose}

Our strategy of evidence-based antibiotic dosing was effective in $85.8 \%$ of patients. In present series, SSI was $9.2 \%$ but other 9 patients who were having minor wound complications required to extend antibiotics multiples dose even if they were not qualified to criteria of SSI which prevented anxiety of surgeon about SSI. Criteria for SSI are well-defined and guidelines for prevention of SSI are well-established. Isolation of microorganism or purulent discharge from operative wound without isolation of the organism qualifies for SSI. Local symptoms of redness and signs like increased local tenderness and heat or swellings are not supposed to be SSI, unless it requires deliberate opening of surgical wound. ${ }^{10}$

Prime importance must be given to these signs or symptoms for continuation of antibiotic, and antibiotic must be discontinued in the absence of these signs or symptoms or these are not exaggerated. Cephalosporins with sulbactam are suitable antibiotics for prophylaxis in clean surgeries for targeting common pathogens responsible for SSI. There is level I evidence which is based on class I data (prospective randomized controlled trials) and strong class II data that single-dose antibiotics given 1 hour prior to incision is equally effective as 5 days antibiotic therapy. Complicated or dirty wounds must be treated with additional doses of appropriate antibiotics. Whereas level II evidence, based on class II data (prospective clinical study or retrospective analysis of reliable data), suggests that majority of clean surgery do not require continuing antibiotics more than 24 hours; unnecessary timing of dose affects efficacy; and duration of surgery more than 3 hours requires additional dose of antibiotics. Prolonged use of broad spectrum antibiotics is inappropriate and increases the cost of treatment with exposing patients to more adverse effects of drug and promotes resistant bacterial strains development. ${ }^{11}$

In clean and clean contaminated procedures, most of the researchers observed that single dose is equally effective rather more effective than three-dose therapy or 5 days therapy. There is no statistical effectiveness of 5 days chemotherapy over three-dose chemotherapy, ${ }^{12}$ even in clean contaminated and contaminated surgeries three doses of antibiotics are effective in controlling SSI, which was 9.6 and $6.7 \%$ in single-dose and three-dose therapy respectively. ${ }^{13}$

\section{Associated Comorbidities and Outcome}

Number of comorbidities like diabetes mellitus, obesity, altered immune response, and increasing age poses patients at risk of developing SSI. Though different hypothesis for SSI are related for different comorbidities, ${ }^{6}$ interestingly, there are common guidelines for prevention of SSI, which can be easily followed. There should be strict implementation of preventive protocol like timing of preparation of operative site just before surgery as well as the method, i.e., hair clipping is preferred over razor shaving in prior night. Minimal preoperative hospital stay, correct and effective selection of antiseptic solution like chlorhexidine-alcohol solution are key factors to 
reduce SSI; apart from careful selection of antibiotics and its administration 1 hour before incision. ${ }^{2}$

In the present study, patients with commonly performed operative procedures and commonly encountered comorbidities were enrolled with effective outcome of $76.19 \%$ in hypertension, which clearly projects that compliance to guidelines and stringent watch on patient's local or systemic signs and symptoms reduces number of doses of antibiotics. It indicates that most patients with comorbidities can be effectively managed with our policy and spared from financial burden.

Of the total diabetic cohort, $57.14 \%$ of diabetes patients were effectively spared of financial burden in the present study, although diabetes mellitus is an independent risk factor for increased SSI in all types of surgery. Though ideal level of plasma sugar is unknown to reduce SSI, patients with known diabetes or patients having perioperative hyperglycemia are equally exposed to increased risk to surgical wound infection. ${ }^{14}$ Patients with blood sugar level above $140 \mathrm{mg} \%$ are at increased risk of SSI and the risk is directly proportional to blood glucose levels above $200 \mathrm{mg}$ \% level, irrespective of long-term control of glycemic status assessed by hemoglobin A1c. Maintaining normothermia intraoperatively and 60 minutes perioperatively plays an important role in reducing SSIs. It is suggested that hypothermia impairs neutrophil function and induces subcutaneous vasospasm. ${ }^{14,15}$

Though our sample size of obese patients is very limited, more than $70 \%$ of the patients had SSI. Most of the present studies depict similar results of increased SSI. An increased rate of SSI is multifactorial, low oxygen tension at surgical site due to hypoperfusion, leading to ischemia, association of obesity to insulin resistance, hyperglycemic episodes perioperatively, longer operative time, and increased tissue dissection are issues for increased SSI. Patients with obesity need increased dose of prophylactic antibiotics. ${ }^{16}$

There is overexpression of collagen VI, elevated levels of 11f3-hydroxysteroiddehydrogenase type I, which is responsible for suppressed angiogenesis and hypoxia of tissue, low oxygen tension in surgical wound, leading to defective collagen synthesis due to ineffective hydroxylation of proline to lysine causing impaired wound healing. Venous insufficiency, imbalanced proinflammatory cytokines, and tissue edema are other factors correlated with impaired wound healing. ${ }^{17}$

\section{CONCLUSION}

Evidence-based randomization of surgical procedures of $<1$ hour duration in clean patients depending on extent of dissection to flexible dose therapy was effective in $85.7 \%$. Strict implementation of preventive guidelines are mandatory to decrease the financial burden on patients or government. Similarly patients are not exposed to more adverse effect of antibiotics without altering the outcome of surgery. Treating doctor will have flexible protocol for antibiotic therapy, depending on the severity of developing complications as per local signs or symptoms and mental satisfaction.

\section{CLINICAL SIGNIFICANCE}

Treatment cost of the therapy will be curtailed with peace of mind and will reduce anxiety about theater environment and asepsis-related issues if doses are modified as per the local sign and symptoms or systemic effects. There may be positive attitude due to liberty of extending the doses of antibiotics rather than adhering to single dose, three doses, or traditional multidose therapy.

\section{REFERENCES}

1. Bratzler DW, Dellinger EP, Olsen KM, Perl TM, Auwaerter PG, Bolon MK, Fish DN, Napolitano LM, Sawyer RG, Slain D, et al. Clinical practice guidelines for antimicrobial prophylaxis in surgery. Am J Health Syst Pharm 2013 Feb;70(3):195-283. Available from: www.ashp.org/surgical-guidelines.

2. SIGN publication no. 104. 2008. [cited 2008 Jul]. Available from: http://www.sign.ac.uk.

3. Tan JT, Coleman K, Norris S, Mapari J, Shastri S, Metz L. Surgical site infection in India: a systematic review of the incidence and economic burden. Value Health 2010 Nov;13(7): A546-A547.

4. Shetty NH, Nagaraja MS, Nagappa DH, Giriyaiah CS, Gowda NR, Laxmipathy Naik RD. A study on surgical site infection (SSI) and associated factors in government tertiary care teaching hospital in Mysore, Karnataka. Int J Med Public Health 2014 Apr-Jun;4(2):171-175.

5. Naik G, Deshpande SR. A study on surgical site infections caused by staphylococcus with special search for methicillin resistant isolates. J Clin Diagn Res 2011 Jun;5(3):502-508.

6. Singh R, Singla P, Chaudhary U. Surgical site infection: classification, risk factors, pathogenesis and preventive management. Int J Pharma Res Health Sci 2014 Jun;2(3):203-214.

7. Talbot TR, Schaffner W. Relation between age and the risk of surgical site infection: a contemporary reexamination of classic risk factor. J Infect Dis 2005 Apr;191(7):1032-1035.

8. Kumar AR. Antimicrobial sensitivity pattern of staphylococcus aureus isolated from pus from tertiary care hospital, Surendranagar Gujarat and issues related to the rational selection of antimicrobials. Sch J App Med Sci 2013;1(5):600-605.

9. Himanshu R, Kumar Vinay BM, Sharadamma KC, Radhakrishna PM. Comparative study of antimicrobial activity of Ceftriaxone in combination with sulbactam and tazobactam using disc diffusion method. IRJP 2012 May;3(5): 331-334.

10. Available from: https://www.cdc.gov/nhsn/pdfs/ pscmanual/9pscssicurrent.pdf.

11. Available from: www.surgicalcriticalcare.net/Guidelines/ antibiotic_prophylaxis.pdf.

12. Surahio AR, Khan AA, Farooq MU, Fatima I. Single versus 3-dose antibiotic prophylaxis in clean and clean contaminated 
operations. J Ayub Med Coll Abbottabad 2010 Oct-Dec; 22(4):91-94.

13. Abro AH, Pathan AH, Siddiqui FG, Syed F, Laghari AA. Single dose versus 24-hours antibiotic prophylaxis against surgical site infections. JLUMHS 2014;13(1):27-31.

14. Latham R, Lancaster AD, Covington JF, Pirolo JS, Thomas CS Jr. Association of diabetes and glucose control with surgical site infection among cardiothoracic surgery patients. Infect Control Hosp Epidemiol 2001 Oct;22(10):607-612.
15. Ata A, Lee J, Bestle SL, Desemone J, Stain SC. Postoperative hyperglycemia and surgical site infection in general surgery patients. Arch Surg 2010 Sep;145(9):858-864.

16. Anaya DA, Dellinger EP. The obese surgical patient: a susceptible host for infection. Surg Infect (Larchmt) 2006 Oct;7(5):473-480.

17. Pierpont YN, Dinh TP, Emerick Salas R, Johnson EL, Wright TG, Robson MC, Payne WG. Obesity and surgical wound healing: a current review. ISRN Obes 2014 Feb;2014:1-13. 